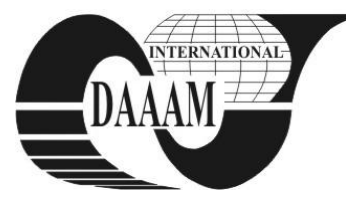

\title{
THE INFLUENCE OF MAGNETIC PULL ON THE NATURAL FREQUENCY OF THE ROTOR OF THE GENERATOR
}

\author{
DONAT, M[artin] \& NAVRATIL, P[etr]
}

\begin{abstract}
The magnetic stiffness of the air gap is obtained as a function of the deflection of the rotor of the generator. This dependence is approximated by a power series. The influence of the unbalanced magnetic pull on the natural frequency of the rotor of the generator is studied by harmonic balance method. Key words: rotor, unbalanced magnetic pull, amplitudefrequency response, harmonic balance method
\end{abstract}

\section{INTRODUCTION}

The magnetic fields, in the air gap between the stator and the rotor of the rotating electric machines, induce electromagnetic forces, which act on the machine structure. Non-uniform thickness of the air gap, caused for example by static eccentricity (eccentrically mounted rotor due to stator), dynamic eccentricity or geometric deviations of the stator and the rotor from ideal cylindrical shape, causes the resultant electromagnetic force is not zero and is called unbalanced magnetic pull (UMP). UMP in electrical rotating machines affects the natural frequencies of the rotor of the machine.

In (Lundström \& Aidanpää, 2006) a formula for calculation a natural frequency of the rotor of the generator, for the case when the perturbation of the air gap is less than $10 \%$ is published. If the eccentricity of the rotor is larger than $10 \%$, the non-linear effects of the UMP will affect the results. To study the influence of the UMP on the natural frequencies of the rotor for this case a simple method was designed. This method is based on an approximation of results obtained by numerical calculation and harmonic balance method.

\section{METHODS}

A computational model used in this study consists of the de Laval rotor on nonlinear anisotropic supports and model of UMP for machines with one pole pair, presented by (Guo et al., 2002).

$$
\begin{gathered}
m \ddot{x}+\left(2 b_{s x}+b_{s u r}\right) \dot{x}+2 k_{s x}\left(1+\mu_{s x} x^{2}\right) x \\
=m a \Omega^{2} \cos (\Omega t)+F_{P x} \\
m \ddot{y}+\left(2 b_{s y}+b_{s u r}\right) \dot{y}+2 k_{s y}\left(1+\mu_{s y} y^{2}\right) y \\
=m a \Omega^{2} \sin (\Omega t)-m g+F_{P y} \\
F_{P x}=f_{1} \cos (\Omega t)+f_{2} \cos (2 \omega t-\Omega t)+f_{3} \cos (2 \omega-3 \Omega t) \\
F_{P y}=f_{1} \sin (\Omega t)+f_{2} \sin (2 \omega t-\Omega t)-f_{3} \sin (2 \omega t-3 \Omega t) \\
f_{1} \approx \frac{R L \pi \mu_{0} F_{j}^{2}}{\delta_{0}^{2}}\left(\frac{1}{2} \varepsilon+\frac{5}{8} \varepsilon^{3}\right) \\
f_{2} \approx \frac{R L \pi \mu_{0} F_{j}^{2}}{\delta_{0}^{2}}\left(\frac{1}{4} \varepsilon+\frac{5}{16} \varepsilon^{3}\right) \\
f_{3} \approx \frac{R L \pi \mu_{0} F_{j}^{2}}{8 \delta_{0}^{2}} \varepsilon^{3}
\end{gathered}
$$

$$
\varepsilon=\frac{\sqrt{\left(x+x_{\text {stat }}\right)^{2}+\left(y+y_{\text {stat }}\right)^{2}}}{\delta_{0}}
$$

where $m$ is the mass of the rotor, $b_{s x}, b_{s y}$ are the damping coefficients of the supports, $b_{\text {sur }}$ is the damping coefficient of the surroundings, $k_{s x}, k_{s y}$ are the base stiffness coefficients of the supports, $\mu_{s x}, \mu_{s y}$ are coefficients of nonlinearity, $a$ is the mass eccentricity distance, $\Omega$ is the angular frequency of the rotor, $t$ is time, $g$ is the gravitational acceleration, $F_{P x}, F_{P y}$ are components of the UMP, $\omega$ is the frequency of the electricity supplied, $R$ is the radius of the generator rotor, $L$ is the length of the generator rotor, $\mu_{0}$ is the air permeance, $F_{j}$ is the amplitude of the magnetomotive force of the rotor exciting current, $\delta_{0}$ is the mean air gap length, $\ddot{x}, \ddot{y}$ are the accelerations, $\dot{x}, \dot{y}$ are the velocities, $x, y$ are dynamic displacements and $x_{\text {stat }}, y_{\text {stat }}$ are static eccentricity in directions of the coordinate systems. The subscripts of the parameters $x$ and $y$ refer to respective dimensions to the axes of the coordinate system. This computational model was described in detail in (Donát, 2011).

First, were calculated amplitude-frequency responses in directions $x$ and $y$. Equations of motion (1) and (2) were solved numerically in Matlab by solver ODE15s. Parameters used for the calculations are:

$$
\begin{array}{lll}
m=18,15 \mathrm{~kg} & b_{s x}=900 \frac{\mathrm{Ns}}{\mathrm{m}} & \mu_{s x}=2 \cdot 10^{7} \frac{1}{\mathrm{~m}^{2}} \\
R=0,059 \mathrm{~m} & b_{s y}=1000 \frac{\mathrm{Ns}}{\mathrm{m}} & \mu_{s y}=2 \cdot 10^{7} \frac{1}{\mathrm{~m}^{2}} \\
L=0,1551 \mathrm{~m} & k_{s x}=2,5 \cdot 10^{6} \frac{\mathrm{N}}{\mathrm{m}} & \mu_{0}=4 \cdot \pi \cdot 10^{-7} \frac{\mathrm{Vs}}{\mathrm{Am}} \\
a=5 \cdot 10^{-5} \mathrm{~m} & k_{s y}=5 \cdot 10^{6} \frac{\mathrm{N}}{\mathrm{m}} & F_{j}=684 \mathrm{~A} \\
\delta_{0}=2,2 \cdot 10^{-3} \mathrm{~m} & \omega=50 \mathrm{~Hz} & x_{\text {stat }}=5 \cdot 10^{-4} \mathrm{~m} \\
b_{\text {sur }}=819 \frac{\mathrm{Ns}}{\mathrm{m}} & g=9,81 \frac{\mathrm{m}}{\mathrm{s}^{2}} & y_{\text {stat }}=0 \mathrm{~m}
\end{array}
$$

The amplitude-frequency responses were published in (Donát 2011). According to (Sprysl et al., 1996) the magnetic stiffness $K_{P x}$ and $K_{P y}$ of the air gap can be obtained from equations (9). In Figure 1 magnetic stiffness $K_{P x}, K_{P y}$ and stiffness of supports $K_{s x}, K_{s y}$ as function of the angular frequency $\Omega$ are showed.

$$
K_{P x}=-\left|\frac{F_{P x}}{x+x_{s t a t}}\right|, \quad K_{P y}=-\left|\frac{F_{P y}}{y+y_{\text {stat }}}\right|
$$

A comparison, of $K_{s x}-\Omega$ and $K_{P x}-\Omega$ shows, that $K_{P x}-\Omega$ is a piecewise constant in the study area. Natural frequency of the rotor in direction $x$ can thus be calculated from equation (10).

$$
\Omega_{N x}=\sqrt{\frac{k_{s x}\left(1+\frac{3}{4} \mu_{s x} x^{2}\right)-K_{P x i}}{m}-\left(\frac{2 b_{s x}+b_{s u r}}{2 m}\right)^{2}}, i=1,2, \ldots n
$$

Where $n$ is the number of elements of the set of results. 


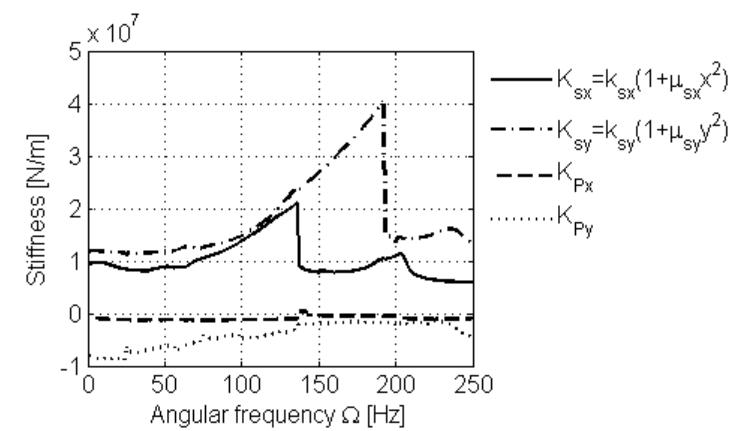

Fig. 1. Stiffness-Angular frequency characteristics

Characteristic of $K_{P y}-\Omega$ is not constant, therefore was obtained dependence $K_{P y}-\mathrm{y}$, see Figure 2 . This dependence has three sections. Section number 1 is area under the resonance and sections 2 and 3 are the areas over the resonance. Notice, dependence $K_{s y}-\mathrm{y}$ in this figure is used for a comparison

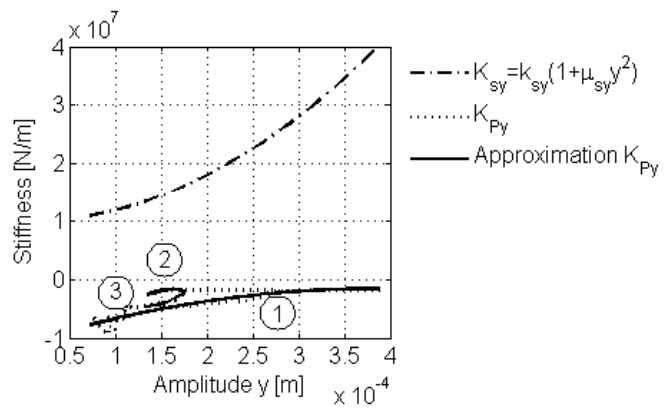

Fig. 2. Stiffness as a function of displacement $y$

Dependence $K_{P y}-$ y can be approximated by equation (11) in section 1 and equation (12) in sections 2 and 3 .

$$
\begin{gathered}
K_{P y}^{*}=k_{n}\left[-1-\mu_{1}\left(y+3.886 \cdot 10^{-4}\right)^{2}\right] \\
K_{P y}^{*}=k_{n}\left(1+\mu_{1} y^{2}+\mu_{2} y^{4}\right)
\end{gathered}
$$

Where $k_{n}$ is a base magnetic stiffness, $\mu_{1}, \mu_{2}$ are coefficients of nonlinearity and $3,886 \cdot 10^{-4}$ is displacement specified by a max amplitude $y$ in section $1 . k_{n}, \mu_{1}$ and $\mu_{2}$ are unknown parameters, which must be determined from approximation. For calculating of the unknown parameters a simple algorithm was used. For example equations (11), there are two unknown parameters in equation (11). From the section 1 of the dependence $F_{P y}-y$ two points were taken and were substituted into equation (11), two equations for two unknown were obtained, from which unknown parameters were calculated. These parameters were substituted back into equation (11), for all $y$ from the section 1 approximation $K_{P y}^{\star}$ of $K_{P y}$ and error of approximation $e$, on this section, were calculated.

$$
e=\sum_{i=1}^{n}\left|K_{P y i}-K_{P y i}^{*}\right|
$$

Where $n$ is the number of elements of the section 1 . This procedure was applied for all possible combinations of elements of the section 1. As a replacement of $K_{P y}$ on section 1 was selected approximation $K_{P y}^{\star}$ with the smallest error $e$.

The natural frequencies of the rotor in direction $y$ can be calculated for example using the harmonic balance method (Schmidt \& Tondl, 1986). This method gives for section 1 equation (14) and for sections 2 and 3 equation (15).

$$
\begin{aligned}
& \Omega_{N y}=\sqrt{\frac{k_{s y}\left(1+\frac{3}{4} \mu_{s y} y^{2}\right)-k_{n}\left[1+\frac{3}{4} \mu_{1}\left(y-3.886 \cdot 10^{-4}\right)^{2}\right]}{m}-\left(\frac{2 b_{s y}+b_{s u r}}{2 m}\right)^{2}} \\
& \Omega_{N y}=\sqrt{\frac{k_{s y}\left(1+\frac{3}{4} \mu_{l y} y^{2}\right)-k_{n}\left(1+\frac{3}{4} \mu_{1} y^{2}+\frac{5}{8} \mu_{2} y^{4}\right)}{m}-\left(\frac{2 b_{s y}+b_{s u r}}{2 m}\right)^{2}}
\end{aligned}
$$

In the Figures 3 are dependencies of natural frequencies $\Omega_{N x}, \Omega_{N y}$ of the rotor on angular frequency $\Omega$. Solid lines in this figures are natural frequencies without considering magnetic stiffness caused by UMP, dashed lines are natural frequencies with considering magnetic stiffness caused by UMP.
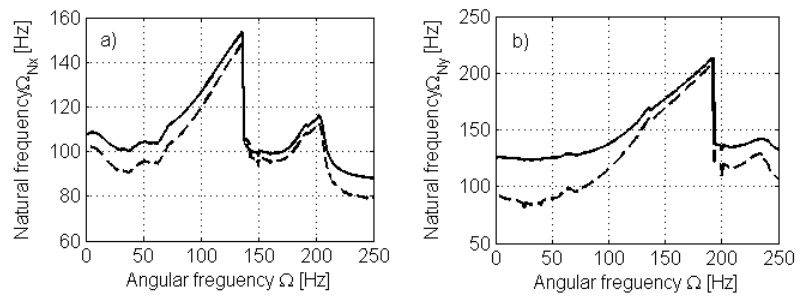

Fig. 3. Natural frequencies as function of angular frequencies

\section{CONCLUSION}

The paper describes a simple method to study the influence of the non-linear UMP on natural frequencies of the rotor of the electric rotating machines based on harmonic balance method. Obtained results confirmed that UMP in electrical rotating machines reduces the natural frequencies of the rotor of the machine. This reduction is more significant when the angular frequency is sufficiently distant from the natural frequency of the rotor of the machine. If the angular frequency is close to the natural frequency of the rotor of the machine, the influence of the UMP on the natural fraquencies of the rotor decreases. This effect is probably caused by non-linear stiffness of the supports of the rotor

The next step will be to study the influence of the nonlinear UMP on natural frequencies of the electric rotating machines for different types of shape deviation of the rotor and the stator.

\section{ACKNOWLEDGEMENT}

This paper was elaborated within the framework of project FSI-S-11-11/1190.

\section{REFERENCES}

Donát, M. (2011). Unbalanced magnetic pull and its effect on dynamic behavior of the rotor with nonlinear anisotropic supports, Proceedings of the $20^{\text {th }}$ DAAAM International Symposium, November 23-26, Vienna, ISSN 1726-9679, ISBN 978-3-901509-83-4

Guo, D.; Chu, F. \& Chen, D. (2002). The unbalanced magnetic pull and its effects on vibration in three-phase generator with eccentric rotor. Journal of Sound and Vibration, Vol. 254, No. 297-312

Lundström, N. \& Aidanpää, J. O. (2004). Dynamic consequence of electromagnetic pull due to deviations in generator shape, Journal of Sound and Vibration, Vol. 301, No. 207-225

Sprysl, H., Vögele \& H., Ebi, G. (1996). The influence of magnetic forces on the stability behavior of large electrical machines, VDI Berichte, Nr.1285

Schmidt, G. \& Tondl, A. (1986).Non-linear vibrations, Cambridge university press, ISBN 978-0-521-26698-7, New York 